\title{
Direct observation of structural dynamics upon photo-excitation in a spin crossover crystal with femtosecond electron diffraction
}

\author{
Yifeng Jiang ${ }^{1}$, Lai Chung Liu ${ }^{2}$, Henrike M. Müller-Werkmeister ${ }^{3}$, Cheng Lu $^{2}$, Dongfang \\ Zhang $^{1}$, Ryan L. Field ${ }^{2}$, Antoine Sarracini ${ }^{1}$, Gustavo Moriena ${ }^{2}$, Eric Collet $^{4}$, and R. J. \\ Dwayne Miller ${ }^{1,2, a}$ \\ ${ }^{1}$ Max Planck Institute for the Structure and Dynamics of Matter, Luruper Chaussee 149, 22607, \\ Hamburg, Germany \\ ${ }^{2}$ Departments of Chemistry and Physics, University of Toronto, 80 St. George Street, Toronto, \\ Ontario, M5S 3H6, Canada \\ ${ }^{3}$ Institute of Chemistry, University Potsdam, Karl-Liebknecht-Strasse 24-25, 14476 Potsdam, \\ Germany \\ ${ }^{4}$ Institute de Physique de Rennes, UMR 6251 UR1-CNRS, Bat 11A Campus de Beaulieu, University \\ Rennes 1, Rennes, France
}

\begin{abstract}
Photoinduced spin transitions are studied by femtosecond electron diffraction to understand ultrafast structural dynamics associated with intersystem crossing. The results indicate the structural reorganization occurs within $2.3 \mathrm{ps}$, as the metal-ligand bond distribution narrows during intramolecular vibrational energy redistribution.
\end{abstract}

\section{Introduction}

Photoinduced spin crossover (SCO) dynamics undergo extensive electronic spin transitions and distortions of the molecular structure with unit quantum yield on femtosecond timescales. These SCO complexes can trigger spin crossover (SCO), a reversible electronic transition between a low-spin (LS) to a high-spin (HS) state that can be induced by applied pressure, temperature, magnetic fields, or light irradiation $[1,2]$. The photoinduced SCO from the LS state to the HS state is dipole forbidden and cannot be directly excited optically. The spin transition occurs through a series of radiationless processes that coupled the states of different spin multiplicity: typically, the initial singlet state is excited by visible light to a singlet metal-to-ligand charge transfer $\left({ }^{1} \mathrm{MLCT}\right)$ state; $\mathrm{a}^{1} \mathrm{n}$ intersystem spin transition occurs to a higher spin state followed by a relaxation cascade from the initial Franck-Condon modes connecting the two surfaces to potential minimum of the HS surface. What are the modes and can we modify them to control spin dynamics?

Cis-bis(thiocyanato)-bis(N-2'-pyridyl methylene)-4-(phenylazo) aniline iron(II)

\footnotetext{
${ }^{1}$ Corresponding author: dwayne.miller@mpsd.mpg.de
} 
$\left(\left[\mathrm{Fe}(\mathrm{PM}-\mathrm{AzA})_{2}\right](\mathrm{NCS})_{2}\right)$ is a system for $\mathrm{SCO}$ in $\mathrm{Fe}(\mathrm{II})$ metal compounds [3,4] (Fig. 1a). The photoinduced SCO process in single crystalline $\left[\mathrm{Fe}(\mathrm{PM}-\mathrm{AzA})_{2}\right](\mathrm{NCS})_{2}$ has been identified by femtosecond optical pump-probe reflectivity studies and XANES [3,4] as a two-step ISC process with a fast $(<150 \mathrm{fs})$ relaxation of short-lived intermediate states (INT) followed by vibrational cooling of the subsequent HS state within 1.5-2 ps. All static crystallographic studies [3-5] point to an $\mathrm{Fe}-\mathrm{N}$ bond elongation by $\sim 0.2 \AA$ in the HS compared to the LS state, caused by the transfer of two electrons to the antibonding $\mathrm{e}_{\mathrm{g}}$ orbitals and the loss of $\pi$-backbonding which weaken the Fe-N bonds. Despite considerable efforts, the precise nature of the structural dynamics remains elusive, especially in the solid state.

\section{Experimental Setup}

Here, we tackled this problem by studying a SCO material with femtosecond electron diffraction (FED). The enhanced sensitivity of FED allows us to directly probe atomic positions within a crystal lattice and resolve all the relevant nuclear motions during SCO [6]. The sensitivity of electron diffraction to the key motions involved can be confirmed by taking advantage of the temperature dependence to go between LS and HS structures. FED experiment was performed with excitation conditions similar to optical pump-probe reflectivity studies (Fig. 1b). Samples were kept at $170 \mathrm{~K}$, photoexcited by $60 \mathrm{fs} \mathrm{FWHM}$ pulses, centered at $800 \mathrm{~nm}$ and monitored by 270 fs FWHM electron source. At 1.28 $\mathrm{mJ} / \mathrm{cm}^{2}$ excitation fluence, there is no damage over the duration of the experiment.

a)

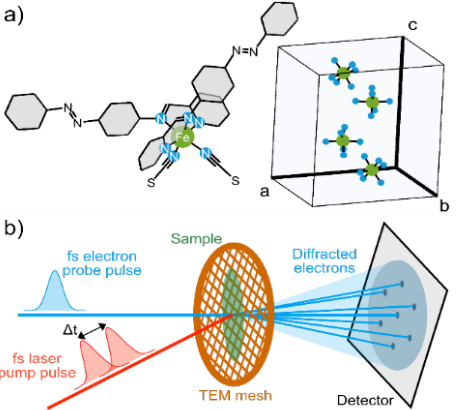

Fig. 1. a) Low-spin molecular structure of $\left[\mathrm{Fe}(\mathrm{PM}-\mathrm{AzA})_{2}\right](\mathrm{NCS})_{2}$; arrangement of the $\mathrm{FeN}_{6}$ complexes within the crystallographic unit cell. b) Schematic of our femtosecond electron diffraction setup [7].

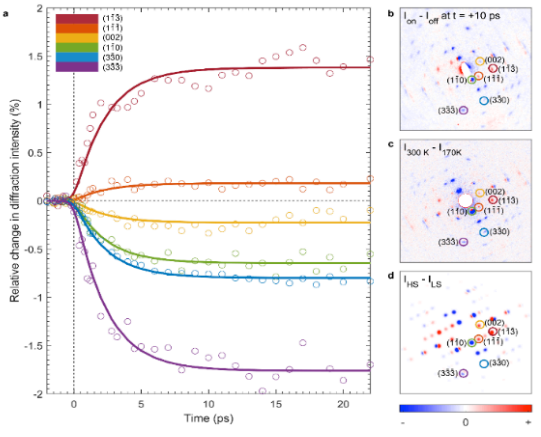

Fig. 2. a) Short-time relative change in the intensity of select Bragg spots from $t=-5$ to 22 ps. b) signal induced by photoexcitation, measured after 10 ps time delay; c) difference between LT and HT data; d) difference between LT and HT data, simulated using static X-ray diffraction structures [7].

\section{Result and Discussion}

By monitoring the intensity of the time-varying Bragg reflections (Fig.2), we observed the formation of a photoinduced structure similar to that of the thermally induced HS state. The data and supporting refinement calculations indicate that the SCO structural reorganization occurs within 2.3 ps. The time constant matches the timescale of IVR observed in previous transient UV-VIS spectroscopy work. Recent XANES work suggests metal ligand bond 
distribution after photoexcitation narrows on the same timescale as spectral features associated with IVR. Our results are consistent with this picture and provide direct observation of the IVR from the initially created hot excited HS molecules and energy transfer from metal ligand stretching motions to the rearrangements of the ligands and the unit cell(Fig.3). The structural dynamics involve collectively the metal-ligand bond elongation, ligand butterfly motion, and unit cell expansion. These global changes were all found to share the same time constant (2.3 ps) (Fig. 4). The structural dynamics can be fully modelled by three key modes, such that the atomic motions induced by IVR after SCO are monitored with ultrafast temporal resolution and atomic spatial resolution. The picture that emerges is that of a few strongly coupled modes excited during the initial Franck-Condon (FC) transition, which dominate the relaxation process towards the HS state, ultimately giving net elongation of the $\mathrm{Fe}-\mathrm{N}$ bond. The relaxation pathway from the initial FC weighted spin transition to the final structure of the photoexcited HS state has been determined, which now provides atomic level detail on recent x-ray spectroscopic results for a fully consistent picture. These results show that the nuclear dynamics definitively proceed on a much different time scale than the electronic dynamics and posit that the initial FC crossing involves higher frequency vibrational modes than the previously thought Fe-N motions [7].

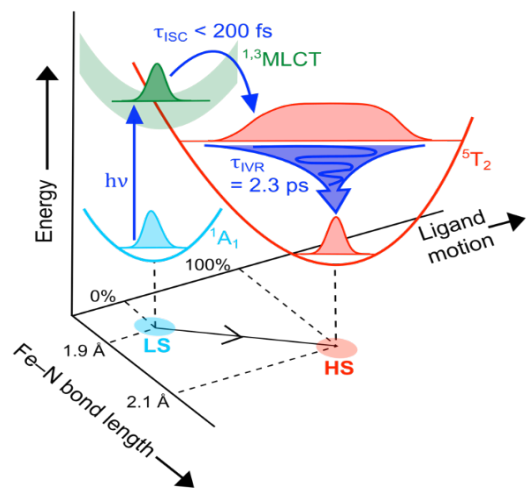

Fig. 3. Schematic representation of the proposed reaction pathway of $\mathrm{SCO}$ in configuration space [7].

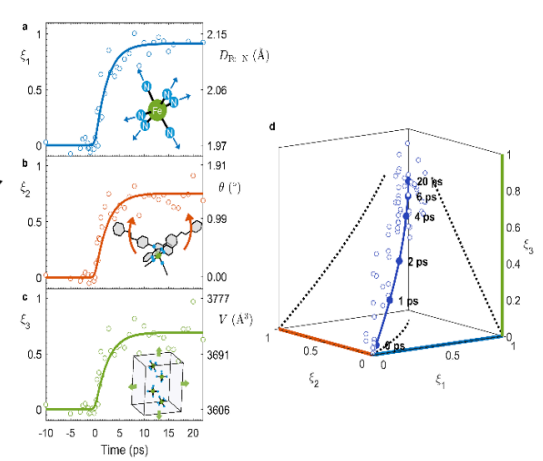

Fig. 4. Molecular movie. a)-c), Timedependence of refined reaction coordinates. d), Plot of independent refinement over reaction-coordinate space [7].

\section{References}

[1] G. Auböck, and M. Chergui, Nat. Chem. 7, 629 (2015).

[2] A. Hauser, in Spin Crossover in Transmission Metal Compounds II, Gütlich, P. \& Goodwin, H. A eds. 155 (Topics in Current Chemistry 234, Springer, 2004).

[3] A. Marino, et al. Polyhedron 66, 123-128 (2013).

[4] M. Cammarata, M. et al. Phys. Rev. Lett. 113, 227402 (2014).

[5] A. Marino, et al. Struct. Dyn. 3, 023605 (2016).

[6] M. Gao et al, Nature, 496, 343. (2013).

[7] Y. Jiang, et al., Angew. Chem. Int. Ed. 56, 7130 (2017). 